\title{
An attributive component of the condition of people with the diagnosis of "Ischemic heart disease. Effort angina".
}

\author{
Nataliya A. Kasimovskaya ${ }^{1}$, Eleonora V. Fattakhutdinova², Anvar N. Khuziakhmetov ${ }^{3}$, Nikolay G. Kutlin ${ }^{4}$, Yulia A. Kostina ${ }^{5}$, \\ Igor G. Kochetkov ${ }^{6}$
}

\begin{abstract}
Objective: The article is devoted to the study of attributive components of people diagnosed with Ischemic heart disease. Effort angina. The results of empirical studies of the attributive process are discussed in the article. Now more and more researchers, both domestic and foreign, are beginning to consider attribution as one of the principles of human existence. Attributive processes initially began to be developed in line with the cognitive orientation of social psychology. Currently, they are actively considered in the framework of the psychology of social cognition.

Method: We conducted a study on 24 subjects with an average cholesterol level of $7.426 \mathrm{mmol} /$ I (concomitant diseases - hypertension, diabetes, obesity, metabolic syndrome), who underwent treatment in the Central City Clinical Hospital in the rehabilitation department with the diagnosis of Ischemic Heart Disease. Effort Angina. Initially, we conducted the survey of subjects in order to identify their subjective judgment about the cause of the disease. They were asked a question: "In your opinion, what is the cause of your heart disease?"

Results: The results of the survey conducted testify that only a small percentage of the subjects believe that they could exert any influence on their health and, for example, to reduce environmental or biological factors. One would assume that such results may be a specific demonstration of the fact that an individual gains experience that it is precisely human health that is difficult to correct and change and simply does not solve it due to the complexity of the problem. However, data obtained in the course of other experiments and studies [19] show that a person is inclined to explain what is happening by circumstances or situational causes, rather than personal ones. The results obtained with the help of T. Dembo's cognitive selfassessment technique indicate that women believe that they actually have much less physical exertion and their daily routine is more correct than people who are close to them, in their opinion, think about it. We can assume that introjects are more often peculiar to women than men that are related to housekeeping, which sometimes requires considerable physical activity and time. When a woman has to, at the insistence of a doctor, reduce her physical activity and somehow comply with the daily regimen, she feels discomfort because of which she believes that she "does nothing at all", although her relatives continue to persuade her to have a rest. Here it is appropriate, in our opinion, to raise the problem of accepting oneself and, as a sequence, to take adequate care of oneself. Significant differences in the self-attributive and reflexive components were also discovered in the emotional sphere of the female subjects. In their opinion, they experience low intensity of negative emotions, such as fear and anger, but at the same time they believe that their close relatives radically disagree. This situation can be explained either by the fact that they assume that they have negative emotions more strongly than they would like (and what they admit to this in the study), or they are judged by the words of relatives who can also make their judgments by interpreting the facts with varying degrees of adequacy.

Conclusion: In conclusion, it should be noted that during the analysis we encountered at least two facts that require further empirical research. Firstly, we identified three types of explanations (attributions) in the subjects' answers: adverbial, subjective and personal, and the latter type of causal attribution is not expressed clearly in the subjects of the study. This poses new questions: does a particular type of situation contribute to this perception, or are there any other factors (including personal ones) playing the role here. Secondly, the following questions arise: how exactly does a subject choose only one alternative from a variety of explanatory reasons. All these questions will be understood as a part of our further research
\end{abstract}

Keywords: causal attribution, self-attribution, ischemic heart disease, effort angina, myocardial infarction with ST segment elevation, cognition

\section{INTRODUCTION}

Now more and more researchers, both domestic and foreign, are beginning to consider attribution as one of the principles of human existence $(1,2)$. Attributive processes initially began to be developed in line with the cognitive

\footnotetext{
Sechenov First Moscow State Medical University, Moscow, Russia

2 Kazan State Medical University, Kazan, Russia.

3 Kazan (Volga region) Federal University, Kazan, Russia.

4 Birsk Branch of Bashkir State University, Birsk, Russia.

5 Ogarev Mordovia State University, Saransk, Russia.

6 Ulyanovsk State University, Ulyanovsk, Russia.
}

Correspondence: Igor G. Kochetkov

Ulyanovsk State University, Ulyanovsk, Russia.

E-mail: ikochetkov@yandex.ru

Received: 6 Jun 2018, Accepted: 6 Oct 2018

(C) 2018 by the authors; licensee Modestum Ltd., UK. This article is an open access article distributed under the terms and conditions of the Creative Commons Attribution License (http://creativecommons.org/licenses/by/4.0/).

Electronic Journal of General Medicine 
orientation of social psychology. Currently, they are actively considered in the framework of the psychology of social cognition $(3,4)$.

In our work, we rely on the research of a number of authors. Our assumption is that, being in an identical situation, a specific subject evaluates and perceives the events in his/her own way. Gulevich in their abstractive review, relying on the works of foreign authors, give the following definition of attribution, which has already become generally accepted, and describe several types of this concept, which have already become classical: causal attribution, self-attribution, spontaneous attribution, emotion attribution, responsibility attribution, social attribution. An attributive process is understood as attributing characteristics to social objects that are not directly represented in the field of perception (4).

Modern American and European researchers are also studying attribution in different attribute areas.

Thus, the general laws of the formation of causal attribution and self-attribution are studied. Quiamzade and L'Huillier (5) studied the situations when dummy subjects performed extremely unusual actions with investments (these manipulations are often very significant for a Western person), and how these actions were copied by others. These researchers, having conducted a series of experiments, found that, firstly, the initiator of the movement is an expert who has the advantage that the others follow him in his decision to invest, compared with the initiator who is not an expert. It was also found that such an advantage arises only when the decisions of the initiator are out of the ordinary and even deviate from the norm. Secondly, the researchers proved that when people see the same extraordinary investments, but do not know whether the initiator has confidential information or not, they follow him just as if he possessed such information.

Thus, the study showed that people believed that the unknown initiator had confidential information (that is, they attributed some knowledge to him and explained his behavior in some way). Moreover, following the unknown initiator's example was statistically indistinguishable from the one about which it was precisely known that he had confidential information.

Mason and Morris (6) examined the role of attributive aspects of automatism in the cultural environment by the example of residents of the West and Asia using the neurological approach. The fundamental task, in their opinion, is to identify the causes underlying behavior of other people. Their data suggest that residents of East Asia, when perceiving more often than residents of the West, refer to the social context in explaining the causes of actions of other people.

Mason and Morris (6) also considered that some data from social cognitive neurological research would contribute to a deeper consideration of this problem. In these authors' opinion, the social approach of neurology can be used to identify whether culture affects automatic, controlled, or both types of attributive processes. As this review has shown, neurologists are just beginning to accumulate evidence on the issue of automatism. For example, in the socioneurological literature there are no attempts to identify areas of the brain that are activated when participants deliberately explain behavior of another person from the point of view of social constraints. Scientists believe that a clearer understanding of attributive processes will occur when social neurologists take account of the relevant sociopsychological data and include paradigms in their research, which are used by traditional researchers of attributive processes. Although Mason and Morris emphasized the evidence that culture affects the automatic processes of this concept; they also assert that culture most likely affects both automatic and controllable elements (7-10).

At present, researchers also have proposed a number of applied tasks of studying the attribution of causes and results of a subject's activity $(2,11-15)$.

In domestic psychology, Yurevich was engaged in the analysis of causal explanations (16-18). Proceeding from the data indicating the rigidity and stability of the attribution process, as well as the multifunctional nature of this process, he expressed an idea of the functioning of the reference causality schemes learned in the process of socialization. Yurevich identifies three levels of mastering these schemes. Firstly, they arise from the concept of causality, and are fixed at the level of society as a whole. Secondly, the standards of causality can be fixed at the level of individual groups in certain group values and norms. Thirdly, causality schemes can be developed by a person independently, in the process of forming individual experience, thereby supplying situations with different explanations depending on the individual experience of a person.

In contemporary Russian research Vodyakha identifies three criteria for the analysis of attribution and self-attribution: the subject of attribution (what exactly is being explained), its object (whose actions are subject to explanation) and the nature of the information attributed (what is being attributed) (19). The author believes that the phenomenon of selfattribution allows one to clarify not only the tasks, but also the causes of the results of the activity directly related to the subject, and also correlates with the personal styles of explanation. Thus, the studies and the formation of attributive processes are important research problems of both foreign and domestic authors (20 - 24). 


\section{MATERIALS AND METHODS}

Initially, our scientific interest was focused on the study of attributive processes in various life situations. We have identified causal and self-attributive factors of the subjective perception of a complicated life situation. Further in our study, starting from the work carried out by Emelyanenkova, after analyzing the works of Sedunova and especially Menzorov, we decided to extend the scope of the study of the attribute component to individuals with ischemic heart disease $(25-31)$.

For many years ischemic heart disease has been and is the main cause of mortality in many economically developed countries. Currently, cardiovascular diseases play a crucial role in the evolution of total mortality in Russia.

In 2006, the mortality rate from diseases of the blood circulation system in the Russian Federation was $56.5 \%$ in the overall structure of mortality. Of these, about half are deaths from ischemic heart disease. In the countries of Western Europe, the USA, Canada, Australia over the past decades there has been a steady decline in mortality from this disease.

In Russia, mortality rates of cardiovascular diseases are much higher, but over the last 2-3 years there has been a tendency towards their stabilization. The frequency of angina increases dramatically with age: in women from $0.1-1 \%$ at the age of $45-54$ to $10-15 \%$ at the age of $65-74$; in men from $2-5 \%$ at the age of $45-54$ to $10-20 \%$ at the age of $65-74$. In the majority of European countries, the prevalence of angina is 20-40 thousand people per 1 million of the population.

Currently, practitioners use the classification of coronary artery disease according to clinical forms, recommended in 1979 by a group of WHO experts:

1) Sudden coronary death (primary cardiac arrest):

a) sudden coronary death with successful resuscitation;

b) sudden coronary death (death);

2) Angina pectoris:

a) stable exertional angina (with indication of functional class);

b) coronary syndrome $X$;

c) vasospastic angina;

d) unstable angina;

e) progressive angina pectoris;

f) first-time angina;

g) early post-infarction angina;

3) Myocardial infarction.

4) Cardiosclerosis.

5) painless form of ischemic heart disease.

As we can see from this classification, ischemic heart disease can acutely manifest itself: myocardial infarction or even sudden death, but more often it develops gradually, turning into a chronic form. In such cases, one of its main manifestations is angina pectoris. According to the Framingham study, angina pectoris is the first symptom of ischemic heart disease in men in $40.7 \%$ of cases and in women in $56.5 \%$.

Angina pectoris is caused by transient myocardial ischemia, the basis of which is the disparity between the myocardial oxygen demand and its delivery through the coronary arteries. This disease appears during physical exertion or stressful situations in the presence of the narrowing of the coronary arteries' lumen, as a rule, not less than by $50-70 \%$. The greater the degree of coronary artery stenosis, the more severe angina is. That is, we assume that an individual who is diagnosed with "Ischemic heart disease. Effort angina" will undoubtedly analyze his condition, and, probably, he wonders why this has happened to him.

Our interest is focused on how he will explain the cause of his illness (to determine his type of attribution according to Kelly's classification) and what types of attribution a patient has when analyzing this. 
To do this, we conducted a study on 24 subjects with an average cholesterol level of $7.426 \mathrm{mmol} /$ I (concomitant diseases - hypertension, diabetes, obesity, metabolic syndrome), who underwent treatment in the Central City Clinical Hospital in the rehabilitation department with the diagnosis of Ischemic Heart Disease. Effort Angina.

Initially, we conducted the survey of subjects in order to identify their subjective judgment about the cause of the disease. They were asked a question: "In your opinion, what is the cause of your heart disease?"

\section{RESULTS AND DISCUSSION}

As a result of the survey, we obtained the following answers:

- $54 \%$ of the subjects attributed the cause of their illness to distress, which arose from experiencing difficult life situations (objective difficulties in family life, an incurable and serious illness of loved ones, an unstable situation in the country and the world, etc.), that is, the subjects demonstrate the attribution of circumstances according to Kelly's classification;

- $20.8 \%$ of people demonstrate a situational type of the attributive causal process, in which the cause is a specific situation (for example, a trauma (fracture) or complications after surgery, or the incompetence of a particular doctor); some subjects also call their heredity is the cause of this disease, which can, in our opinion, also be attributed to the situational type of the attributive process, since in this case the subjects do not monitor their health so well and carefully to reduce their hereditary factor, and even vice versa that they think that their fate in terms of health is predetermined;

- $8.3 \%$ of the subjects demonstrate a personal type of attribution, considering that the cause of their disease could be the use of certain types of psychoactive substances (smoking, excessive drinking) or a wrong / an unhealthy lifestyle.

The rest (16.9\%) of the subjects could not determine the cause of ischemic heart disease.

Further in our study, using Dembo's cognitive self-assessment method, we identified a self-attributive component of the condition of the subjects with the diagnosis of Ischemic Heart Disease. Effort Angina, and also revealed some of their judgments after self-analysis. We gave the subjects nine scales (vertical lines $100 \mathrm{~mm}$ long): physical activity, daily routine, meals, life situations, anger, sadness, fear, pleasure and joy. On each vertical line, the subject had to put a bullet point in the place that describes his condition. Then he again on each vertical line had to put a marking in the place that describes how, in the opinion of the subject himself, his relatives think what his state is.

After processing the data obtained with the use of the Mann-Whitney statistical criterion, designed to assess the differences between the two samples, we obtained the following results.

The women-subjects showed significant differences in self-attributive and reflexive components with an error probability of 0.05 on the following scales: physical activity ( 13 at a critical value of 15), daily routines ( 15 at a critical value of 15), feelings of anger and irritation (11,5 at a critical value of 15) and fear, anxiety (14.5 at a critical value of 15). In men, no significant differences in the self-attributive and reflexive components were found.

\section{CONCLUSION}

The results of the survey conducted testify that only a small percentage of the subjects believe that they could exert any influence on their health and, for example, to reduce environmental or biological factors. One would assume that such results may be a specific demonstration of the fact that an individual gains experience that it is precisely human health that is difficult to correct and change and simply does not solve it due to the complexity of the problem. However, data obtained in the course of other experiments and studies (6) show that a person is inclined to explain what is happening by circumstances or situational causes, rather than personal ones.

The results obtained with the help of Dembo's cognitive self-assessment technique indicate that women believe that they actually have much less physical exertion and their daily routine is more correct than people who are close to them, in their opinion, think about it.

We can assume that introjects are more often peculiar to women than men that are related to housekeeping, which sometimes requires considerable physical activity and time. When a woman has to, at the insistence of a doctor, reduce her physical activity and somehow comply with the daily regimen, she feels discomfort because of which she believes that she "does nothing at all", although her relatives continue to persuade her to have a rest. Here it is appropriate, in our opinion, to raise the problem of accepting oneself and, as a sequence, to take adequate care of oneself. Significant 
differences in the self-attributive and reflexive components were also discovered in the emotional sphere of the female subjects. In their opinion, they experience low intensity of negative emotions, such as fear and anger, but at the same time they believe that their close relatives radically disagree. This situation can be explained either by the fact that they assume that they have negative emotions more strongly than they would like (and what they admit to this in the study), or they are judged by the words of relatives who can also make their judgments by interpreting the facts with varying degrees of adequacy.

In conclusion, it should be noted that during the analysis we encountered at least two facts that require further empirical research. Firstly, we identified three types of explanations (attributions) in the subjects' answers: adverbial, subjective and personal, and the latter type of causal attribution is not expressed clearly in the subjects of the study. This poses new questions: does a particular type of situation contribute to this perception, or are there any other factors (including personal ones) playing the role here. Secondly, the following questions arise: how exactly does a subject choose only one alternative from a variety of explanatory reasons. All these questions will be understood as a part of our further research.

\section{ACKNOWLEDGEMENT}

1. The publication has been supported by the "Russian Academic Excellence Project $5-100$ " of the I M Sechenov First Moscow State Medical University (Sechenov University).

2. The work is performed according to the Russian Government Program of Competitive Growth of Kazan Federal University.

\section{REFERENCES}

1. Kelley H. Attribution theory in social psychology. Nebraska Symposium on Motivation. Nebraska. 1967;15:192238.

2. Krasnov VN. Affective spectrum disorders. Moscow: Practical medicine; 2011.

3. Andreeva GM. Psychology of social cognition. Moscow: The Aspect of Press; 2000.

4. Gulevich OA, Bezmenova I.K. Attribution: general presentation, research directions, mistakes. Moscow: Russian psychological society; 1998.

5. Quiamzade, A., L'Huillier, J.P. (2009). Herding by Attribution of Privileged Information. Journal of Behavioral Decision Making. 1981;22:1-19. https://doi.org/10.1002/bdm.608

6. Mason MF, Morris MW. Culture, attribution and automaticity: a social cognitive neuroscience view. Social Cognitive and Affective Neuroscience; 2010. Retrieved from http://scan.oxfordjournals.org/content/early/2010/ 05/11/scan.nsq034.full

7. Aleksandrovsky YuA. Border mental disorders: a guide for doctors. Moscow: GEOTAR-Media; 2007.

8. Boelen PA, Huntjens RJ, van Deursen DS, van den Hout MA. Autobiographical memory specificity and symptoms of complicated grief, depression, and posttraumatic stress disorder following loss. Journal Behav. Ther. Exp. Psychiatry. 2010;41(4):331-337. https://doi.org/10.1016/j.jbtep.2010.03.003 PMid:20394916

9. Kaplan GI, Sadok BJ. Clinical Psychiatry. Moscow: AST; 1994.

10. Kuo JR, Linehan MM. Disentangling emotion processes in borderline personality disorder: physiological and selfreported assessment of biological vulnerability, baseline intensity, and reactivity to emotionally evocative stimuli. Journal Abnorm. Psychol. 2009;118:531-544. https://doi.org/10.1037/a0016392 PMid:19685950 PMCid:PMC4277483

11. Horowitz MJ. Stress response syndromes and their treatment. V.Hamilton \& D.Warburton (Eds.). Human stress and cognition. New York: Wiley; 1980.

12. Nikityuk DB, Miroshkin DV, Bukavneva NS. Clinical and anthropological approaches. Morphological statements. 2008;1(2):56-62.

13. Nikityuk DB, Miroshkin DV. Immune structures with increased physical exertion. Morphology. 2008;133(2):85-90.

14. Shcherbakova OI, Chernykh AK, Balahanova DK, Midova VO. Stress control practices for mental balance improvement in academic sports. Teoriya i Praktika Fizicheskoy Kultury. 2018;9(1):22-23.

15. Tsygankov BD, Dzhangildin YuT, Schetinina EA. Psycho-psychotherapeutic correction of mental disorders resulting from stress. Siberian Journal of Psychiatry and Narcology. 2006;4(1):301-302. 
16. Luchnikova SV, Mikhailova IV. The specificity of the attributes of the cognitive conformity of the "image-I" of students of Suvorov military schools. Bulletin of the University of PAO. 2011;3:72-75.

17. Mikhailova IV. Attribution elements in conformal situations. Theory and practice of social development. 2013;8:144-146.

18. Shcherbakova Ol. Formation managers stress management skills. Modern training and coaching: new opportunities in business and education. Moscow: Russian University of Economics; 2017.

19. Vodyakha YuE. The style of academic self-attribution of psychology students. Pedagogical education in Russia. 2012;2:47-54.

20. Avedisova AS. Psychopharmacotherapy of patients with post-traumatic stress disorder. Journal of Neurology and Psychiatry. 2009;109(12):46-49.

21. Dmitrieva TB, Drozdov FZ, Kogan BM. Clinical neurochemistry in psychiatry. Moscow: RIO SSC SSP them. V.P. Serbian; 1998.

22. Feldner MT, Monson CM, Friedman MJ. Critical Analysis of Approaches to Targeted PTSD Prevention: Current Status and Theoretically Derived Future Directions. Behav. Modif. 2007;31(1):80-116. https://doi.org/10.1177/0145445506295057 PMid:17179532

23. Jind L, Elklit A, Christiansen D. Cognitive schemata and processing among parents bereaved by infant death. Journal Clin. Psychol. Med. Settings. 2010;17(4):366-377. https://doi.org/10.1007/s10880-010-9216-1 PMid:21110073

24. Leontiev AN. Activity. Consciousness. Personality. Moscow: Meaning; 2005.

25. Emelyanenkova AV. Motivation of power in building an individual career strategy. World of Psychology. 2011;4:260-272.

26. Gnedova SB, Igonina AN. Psychological support for students with disabilities: fear of inconsistency. Actual problems of modern education: experience and innovation, materials. 2013;6:240-244.

27. Litvintsev SV, Snedkov AM, Reznik EV. Combat mental injury: a guide for doctors. Moscow: Medicine; 2005.

28. Menzorov MV, Shutov AM, Serov VA, Mikhailova EV. Acute kidney damage in patients with myocardial infarction and the effectiveness of thrombolytic therapy. Cardiology. 2012;52(5): 8-12. PMid:22839579

29. Sedunova AS. Perfectionism and personality self-regulation styles. Theory and practice of social development. 2013;8:127-129.

30. Petrovsky VA. To the understanding of personality in psychology. Questions of psychology. 1981;2:40-46.

31. Rubinstein SL. Fundamentals of general psychology. St.Petersburg: Peter; 1998.

\author{
$\diamond \diamond \diamond \diamond \diamond \diamond \diamond$ \\ http://www.ejgm.co.uk
}

